\title{
Metaphyseal Widening
}

National Cancer Institute

\section{Source}

National Cancer Institute. Metaphyseal Widening. NCI Thesaurus. Code C98274.

A radiologic finding characterized by an increased width of the metaphyseal regions. It is seen in rickets. 